\title{
Cystinosis: electron microscopic evidence of lysosomal storage of cystine in lymph node
}

\author{
A. D. PATRICK AND B. D. LAKE
}

From the Departments of Chemical Pathology and Morbid Anatomy, Institute of Child Health, London

SYNOPSIS Electron microscopic examination of a biopsy specimen of cystinotic lymph node demonstrated that cystine crystals were entirely intracellular, and that the majority, including those of the largest size, were enclosed by intact limiting membranes. Acid phosphatase activity was localized specifically at the periphery of the crystal profiles and the smallest crystal aggregates were contained in osmophilic dense bodies which had the appearance of progressively swollen lysosomes. The implications of the suggested lysosomal distribution of cystine are discussed.

The aetiology of cystine deposition in the reticuloendothelial cells and cornea of patients with cystinosis remains a confusing problem in the understanding of this rare inborn error of metabolism. Theories have proposed that cystine storage may result from a defect in cystine metabolism confined to the specific sites of its accumulation, or that a more widespread failure of tissues to metabolize cystine leads to its increased concentration in the body fluids, with ultimate phagocytic removal by the reticuloendothelial system. No specific enzyme abnormality has yet been demonstrated to account for cystine accumulation; on the contrary, studies (Patrick, 1962) have shown a normal capacity of cystinotic tissues for the degradation of cystine via several metabolic pathways.

Whatever abnormality is found eventually to be responsible for cystine storage, it seems likely, according to present concepts of the function of lysosomes in autophagic and endocytotic processes, that crystals would, at some stage of intracellular existence, be found within lysosomal particles. Evidence suggesting this has now been obtained by electron microscopic examination of a cystinotic lymph node.

\section{METHODS}

For confirmation of a diagnosis of cystinosis in a boy, aged 5 years, an inguinal lymph node was removed under general anaesthesia. Portions of tissue were (a) frozen in hexane at $-80^{\circ} \mathrm{C}$ for histochemistry, and (b) fixed in glutaraldehyde solution $(2.7 \%$ in $0.1 \mathrm{M}$

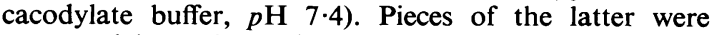
processed by a Gomori procedure to demonstrate acid phosphatase activity, and the remainder, after washing, Received for publication 28 February 1968. was treated with osmic acid and embedded in Araldite by conventional methods. After staining with uranyl acetate, ultrathin sections were examined in a Philips EM 200 electron microscope.

Cryostat sections cut from the frozen tissue were stained with alcoholic basic fuchsin and examined using a polarizer and analyser. The Wollaston test for cystine was applied to air-dried cryostat sections. Introduction of concentrated $\mathrm{HCl}$ under the cover slip effects immediate dissolution of the cystine, followed by the transient crystallization of cystine hydrochloride in the form of needle clusters.

\section{RESULTS AND DISCUSSION}

Crystals, which were mainly short hexagonal prisms and gave a positive Wollaston test, were abundantly distributed throughout cryostat sections (Figs. 1 and 2). The absence of doubly-refractile material in sections prepared for electron microscopy indicated that cystine was dissolved during processing of the tissue. However, except for the largest crystal spaces, around which considerable distortion had occurred, the residual crystal profiles had retained their fine structural detail. Most of these profiles, ranging in size up to approximately $4 \mu$, were delineated by intact membranes (Fig. 3), and were entirely intracellular. Positive reaction for acid phosphatase activity was localized specifically at the periphery of many of the crystal profiles (Fig. 4).

These findings conform to accepted views on the biochemical and morphological identity of lysosomes, and suggest that the cystine crystals were confined within vacuoles derived from the lysosomal system. In cells devoid of cystine deposits, particles which resembled autophagic vacuoles or residual bodies were observed, but no particles resembling 


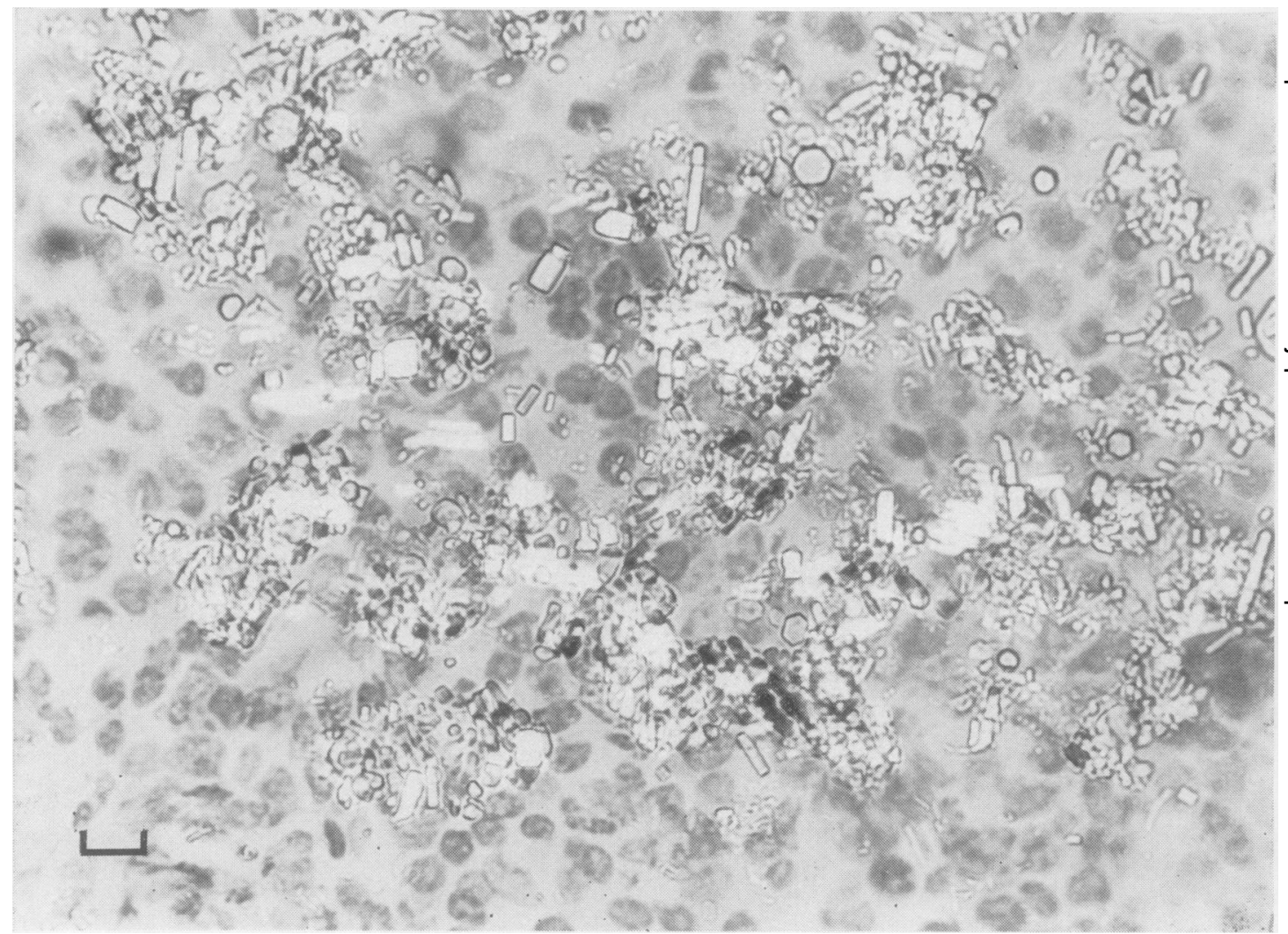

FIG. 1. Cryostat section stained with alcoholic basic fuchsin. Partially polarized light. Scale mark $10 \mu$.

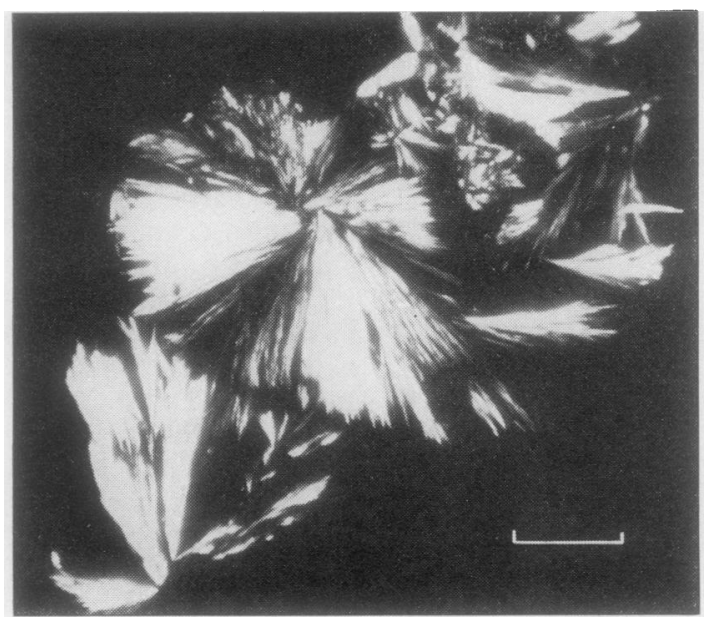

FIG. 2. Wollaston test for cystine. Polarized light. Scale mark $100 \mu$. 


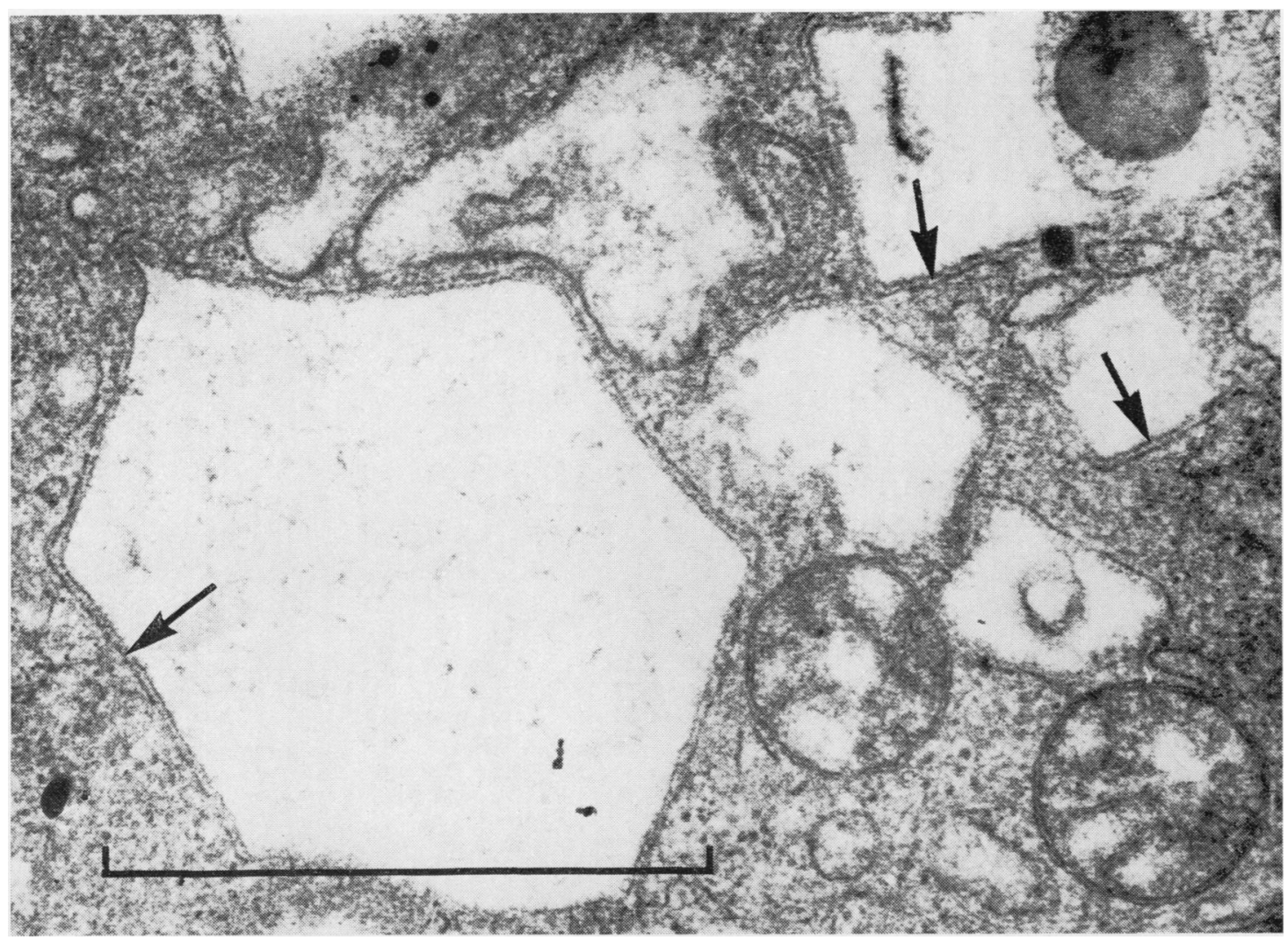

FIG. 3. Electron micrograph showing cystine crystal profiles with intact limiting membranes (arrows). Stained with uranyl acetate, Scale mark $1 \mu$.

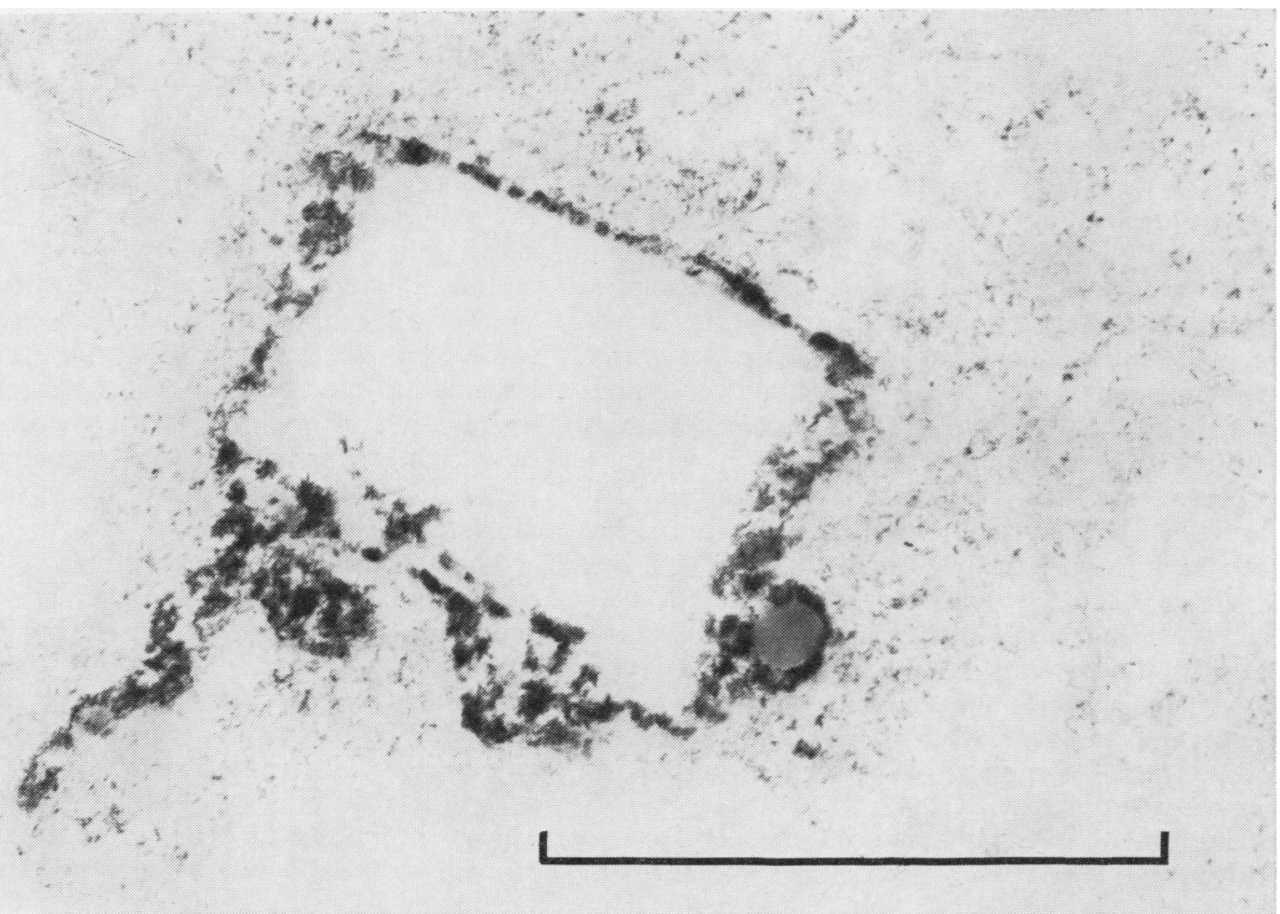

FIG. 4. Electron micrograph showing localization of acid phosphatase activity at the periphery of a cystine crystal profile. Unstained. Lead deposition did not occur in the absence of substrate, $\beta$-glycerophosphate. Scale mark $1 \mu$. 


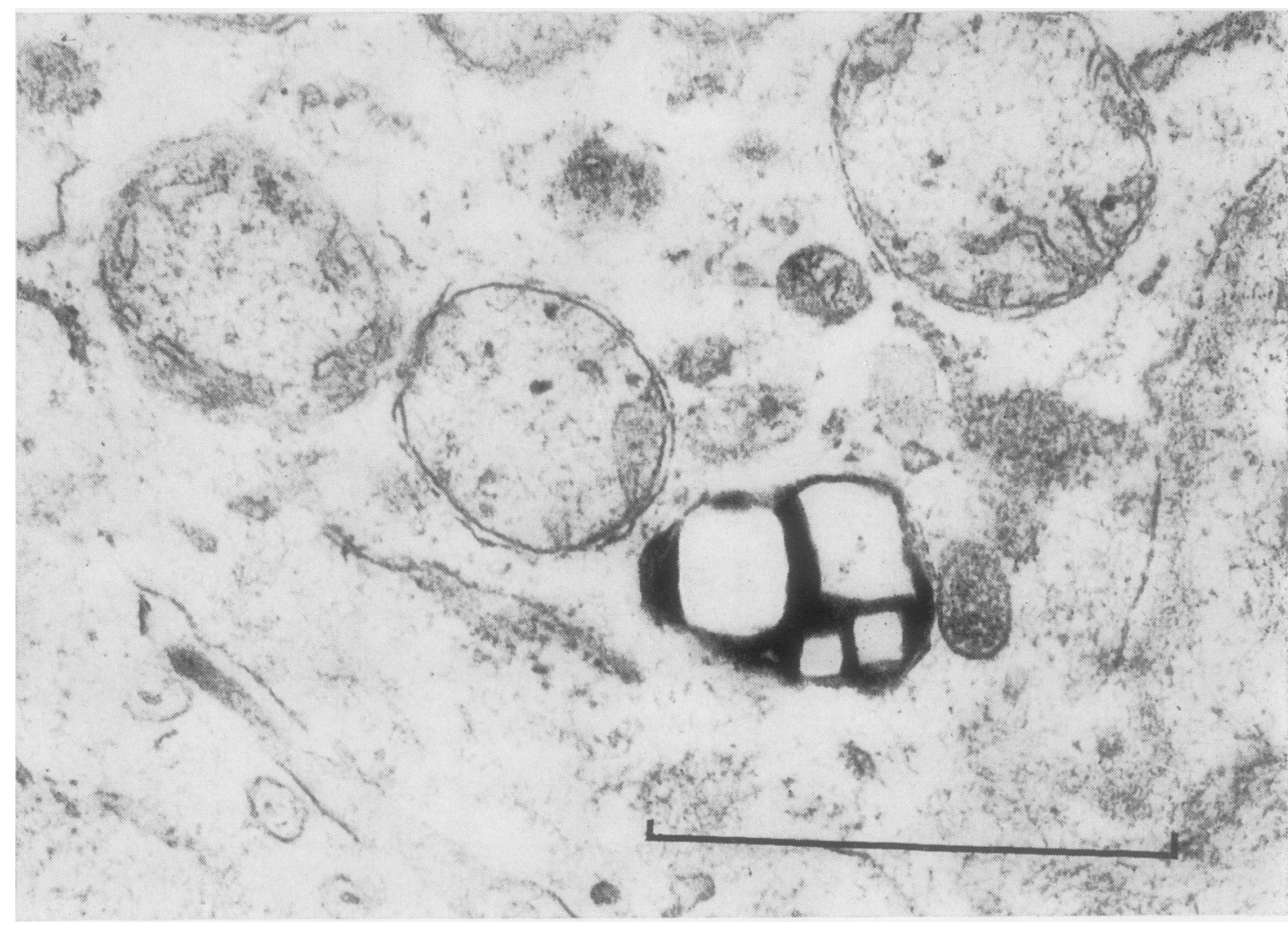

FIG. 5. Electron micrograph showing a group of small crystals within an osmophilic dense body. Stained with uranyl acetate. Scale mark $1 \mu$.

normal lysosomes were seen in cells which contained cystine crystals. In these cells the smallest crystal aggregates were located in osmophilic dense bodies, the largest of which were the size of mitochondria (Fig. 5), and which conceivably represent the earliest stages of intracellular deposition of cystine within the lysosomes. It is suggested that continuous growth of these initiating crystals occurs entirely within the lysosomal compartment, in which a saturated solution of cystine is maintained. The particulate distribution of cystine in cystinosis has also been reported by Schneider, Bradley, and Seegmiller (1967), who found increased concentrations of soluble cystine in peripheral leucocytes of cystinotic patients and their parents, and concluded, from subcellular fractionation studies, that the cystine was compartmentalized within granular components, disruption of which occurred on treatment with Triton-X100.

The possibility that cystinosis results from a primary deficiency of a lysosomal enzyme normally responsible for the degradation of cystine, or from the failure of some other lysosomal process for the elimination or redistribution of cystine residues, arises from the suggested lysosomal storage of cystine. Physical segregation of cystine crystals by the lysosomal membrane would account for their stability in the presence of extralysosomal enzyme systems having a normal capacity for the complete degradation of cystine, and Schneider et al (1967) have rightly stressed the importance of such segregation to the consideration of therapeutic measures aimed at the removal of cystine.

Further implications, with respect to cellular pathology, of abnormal storage of substances by lysosomes have been discussed by Hers (1965). Impaired cell function, including that of normal lysosomal digestive activity, is a possible consequence of progressive storage by the lysosomes, enlargement of which may reach a mechanical limit? at which disruption of the lysosomal membrane allows the release of digestive hydrolases and toxic storage substances into the extralysosomal compartment. Attention has also been drawn to the 
progressivity of lysosomal disorders. After appearing normal for some time after birth, patients develop symptoms of the disease at a rate dependent on the type of substance stored, and on the rate and preferential sites of its accumulation.

Future developments in the study of these aspects of the newly defined lysosomal disorders, of which cystinosis is perhaps a further example, may be expected to provide important contributions to the general understanding of cellular pathology.

\section{REFERENCES}

Hers, H. G. (1965). Gastroenterology, 48, 625.

Patrick, A. D. (1962). Biochem. J., 83, 248.

Schneider, J. A., Bradley, K., and Seegmiller, J. E. (1967). Science, 157, 1321 . 